PROCEEDINGS OF THE

AMERICAN MATHEMATICAL SOCIETY

Volume 138, Number 9, September 2010, Pages 3313-332

S 0002-9939(10)10407-9

Article electronically published on April 30, 2010

\title{
POINT-COFINITE COVERS IN THE LAVER MODEL
}

\author{
ARNOLD W. MILLER AND BOAZ TSABAN
}

(Communicated by Julia Knight)

\begin{abstract}
Let $S_{1}(\Gamma, \Gamma)$ be the statement: For each sequence of point-cofinite open covers, one can pick one element from each cover and obtain a pointcofinite cover. $\mathfrak{b}$ is the minimal cardinality of a set of reals not satisfying $\mathrm{S}_{1}(\Gamma, \Gamma)$. We prove the following assertions:

(1) If there is an unbounded tower, then there are sets of reals of cardinality $\mathfrak{b}$ satisfying $\mathrm{S}_{1}(\Gamma, \Gamma)$.

(2) It is consistent that all sets of reals satisfying $S_{1}(\Gamma, \Gamma)$ have cardinality smaller than $\mathfrak{b}$.

These results can also be formulated as dealing with Arhangel'skiı's property $\alpha_{2}$ for spaces of continuous real-valued functions.

The main technical result is that in Laver's model, each set of reals of cardinality $\mathfrak{b}$ has an unbounded Borel image in the Baire space $\omega^{\omega}$.
\end{abstract}

\section{BACKGROUND}

Let $P$ be a nontrivial property of sets of reals. The critical cardinality of $P$, denoted non $(P)$, is the minimal cardinality of a set of reals not satisfying $P$. A natural question is whether there is a set of reals of cardinality at least non $(P)$, which satisfies $P$, i.e., a nontrivial example.

We consider the following property. Let $X$ be a set of reals. $\mathcal{U}$ is a point-cofinite cover of $X$ if $\mathcal{U}$ is infinite, and for each $x \in X,\{U \in \mathcal{U}: x \in U\}$ is a cofinite subset of $\mathcal{U} \mathbb{1}$ Having $X$ fixed in the background, let $\Gamma$ be the family of all pointcofinite open covers of $X$. The following properties were introduced by Hurewicz [8, Tsaban 19], and Scheepers [15, respectively.

$\mathrm{U}_{\text {fin }}(\Gamma, \Gamma)$ : For all $\mathcal{U}_{0}, \mathcal{U}_{1}, \cdots \in \Gamma$, none containing a finite subcover, there are finite $\mathcal{F}_{0} \subseteq \mathcal{U}_{0}, \mathcal{F}_{1} \subseteq \mathcal{U}_{1}, \ldots$ such that $\left\{\bigcup \mathcal{F}_{n}: n \in \omega\right\} \in \Gamma$.

$\mathrm{U}_{2}(\Gamma, \Gamma)$ : For all $\mathcal{U}_{0}, \mathcal{U}_{1}, \cdots \in \Gamma$, there are $\mathcal{F}_{0} \subseteq \mathcal{U}_{0}, \mathcal{F}_{1} \subseteq \mathcal{U}_{1}, \ldots$ such that $\left|\mathcal{F}_{n}\right|=2$ for all $n$, and $\left\{\bigcup \mathcal{F}_{n}: n \in \omega\right\} \in \Gamma$.

$\mathrm{S}_{1}(\Gamma, \Gamma)$ : For all $\mathcal{U}_{0}, \mathcal{U}_{1}, \cdots \in \Gamma$, there are $U_{0} \in \mathcal{U}_{0}, U_{1} \in \mathcal{U}_{1}, \ldots$ such that $\left\{U_{n}: n \in \omega\right\} \in \Gamma$.

Received by the editors October 21, 2009.

2010 Mathematics Subject Classification. Primary 03E35, 26A03; Secondary 03E17.

${ }^{1}$ Historically, point-cofinite covers were named $\gamma$-covers, since they are related to a property numbered $\gamma$ in a list from $\alpha$ to $\epsilon$ in the seminal paper [7] of Gerlits and Nagy.

(C)2010 American Mathematical Society 
Clearly, $S_{1}(\Gamma, \Gamma)$ implies $\bigcup_{2}(\Gamma, \Gamma)$, which in turn implies $\bigcup_{\text {fin }}(\Gamma, \Gamma)$. None of these implications is reversible in ZFC [19]. The critical cardinality of all three properties is $\mathfrak{b}[2] .2$

Bartoszyński and Shelah 1 proved that there are, provably in ZFC, totally imperfect sets of reals of cardinality $\mathfrak{b}$ satisfying the Hurewicz property $U_{\text {fin }}(\Gamma, \Gamma)$. Tsaban proved the same assertion for $\mathrm{U}_{2}(\Gamma, \Gamma)\left[19\right.$. These sets satisfy $\mathrm{U}_{\text {fin }}(\Gamma, \Gamma)$ in all finite powers [2].

We show that in order to obtain similar results for $S_{1}(\Gamma, \Gamma)$, hypotheses beyond ZFC are necessary.

\section{Constructions}

We show that certain weak (but not provable in ZFC) hypotheses suffice to have nontrivial $S_{1}(\Gamma, \Gamma)$ sets, even ones which possess this property in all finite powers.

Definition 2.1. A tower of cardinality $\kappa$ is a set $T \subseteq[\omega]^{\omega}$ which can be enumerated bijectively as $\left\{x_{\alpha}: \alpha<\kappa\right\}$, such that for all $\alpha<\beta<\kappa, x_{\beta} \subseteq^{*} x_{\alpha}$.

A set $T \subseteq[\omega]^{\omega}$ is unbounded if the set of its enumeration functions is unbounded; i.e., for any $g \in \omega^{\omega}$ there is an $x \in T$ such that for infinitely many $n, g(n)$ is less than the $n$-th element of $x$.

Scheepers [16 proved that if $\mathfrak{t}=\mathfrak{b}$, then there is a set of reals of cardinality $\mathfrak{b}$ satisfying $S_{1}(\Gamma, \Gamma)$. If $\mathfrak{t}=\mathfrak{b}$, then there is an unbounded tower of cardinality $\mathfrak{b}$, but the latter assumption is weaker.

Lemma 2.2 (folklore). If $\mathfrak{b}<\mathfrak{d}$, then there is an unbounded tower of cardinality $\mathfrak{b}$.

Proof. Let $B=\left\{b_{\alpha}: \alpha<\mathfrak{b}\right\} \subseteq \omega^{\omega}$ be a $\mathfrak{b}$-scale; that is, each $b_{\alpha}$ is increasing, $b_{\alpha} \leq^{*} b_{\beta}$ for all $\alpha<\beta<\mathfrak{b}$, and $B$ is unbounded.

As $|B|<\mathfrak{d}, B$ is not dominating. Let $g \in \omega^{\omega}$ exemplify that. For each $\alpha<\mathfrak{b}$, let $x_{\alpha}=\left\{n: b_{\alpha}(n) \leq g(n)\right\}$. Then $T=\left\{x_{\alpha}: \alpha<\mathfrak{b}\right\}$ is an unbounded tower: Clearly, $x_{\beta} \subseteq^{*} x_{\alpha}$ for $\alpha<\beta$. Assume that $T$ is bounded, and let $f \in \omega^{\omega}$ exemplify that. For each $\alpha$, writing $x_{\alpha}(n)$ for the $n$-th element of $x_{\alpha}$ :

$$
b_{\alpha}(n) \leq b_{\alpha}\left(x_{\alpha}(n)\right) \leq g\left(x_{\alpha}(n)\right) \leq g(f(n))
$$

for all but finitely many $n$. Thus, $g \circ f$ shows that $B$ is bounded, a contradiction.

Theorem 2.3. If there is an unbounded tower (of any cardinality), then there is a set of reals $X$ of cardinality $\mathfrak{b}$ that satisfies $S_{1}(\Gamma, \Gamma)$.

Theorem 2.3 follows from Propositions 2.4 and 2.5 .

Proposition 2.4. If there is an unbounded tower, then there is one of cardinality $\mathfrak{b}$.

Proof. By Lemma 2.2, it remains to consider the case $\mathfrak{b}=\mathfrak{d}$. Let $T$ be an unbounded tower of cardinality $\kappa$. Let $\left\{f_{\alpha}: \alpha<\mathfrak{b}\right\} \subseteq \omega^{\omega}$ be dominating. For each $\alpha<\mathfrak{b}$, pick $x_{\alpha} \in T$ which is not bounded by $f_{\alpha} .\left\{x_{\alpha}: \alpha<\mathfrak{b}\right\}$ is unbounded, being unbounded in a dominating family.

\footnotetext{
${ }^{2}$ Blass's survey [4] is a good reference for the definitions and details of the special cardinals mentioned in this paper.
} 
Define a topology on $P(\omega)$ by identifying $P(\omega)$ with the Cantor space $2^{\omega}$, via characteristic functions. Scheepers's mentioned proof actually establishes the following result, to which we give an alternative proof.

Proposition 2.5 (essentially, Scheepers [16]). For each unbounded tower $T$ of cardinality $\mathfrak{b}, T \cup[\omega]<\omega$ satisfies $\mathrm{S}_{1}(\Gamma, \Gamma)$.

Proof. Let $T=\left\{x_{\alpha}: \alpha<\mathfrak{b}\right\}$ be an unbounded tower of cardinality $\mathfrak{b}$. For each $\alpha$, let $X_{\alpha}=\left\{x_{\beta}: \beta<\alpha\right\} \cup[\omega]^{<\omega}$. Let $\mathcal{U}_{0}, \mathcal{U}_{1}, \ldots$ be point-cofinite open covers of $X_{\mathfrak{b}}=T \cup[\omega]^{<\omega}$. We may assume that each $\mathcal{U}_{n}$ is countable and that $\mathcal{U}_{i} \cap \mathcal{U}_{j}=\emptyset$ whenever $i \neq j$.

By the proof of Lemma 1.2 of [6], for each $k$ there are distinct $U_{0}^{k}, U_{1}^{k}, \cdots \in \mathcal{U}_{k}$, and an increasing sequence $m_{0}^{k}<m_{1}^{k}<\ldots$, such that for each $n$ and $k$,

$$
\left\{x \subseteq \omega: x \cap\left(m_{n}^{k}, m_{n+1}^{k}\right)=\emptyset\right\} \subseteq U_{n}^{k} .
$$

As $T$ is unbounded, there is $\alpha<\mathfrak{b}$ such that for each $k, I_{k}=\left\{n: x_{\alpha} \cap\left(m_{n}^{k}, m_{n+1}^{k}\right)=\right.$ $\emptyset\}$ is infinite.

For each $k,\left\{U_{n}^{k}: n \in \omega\right\}$ is an infinite subset of $\mathcal{U}_{k}$, and thus a point-cofinite cover of $X_{\alpha}$. As $\left|X_{\alpha}\right|<\mathfrak{b}$, there is $f \in \omega^{\omega}$ such that

$$
\forall x \in X_{\alpha} \exists k_{0} \forall k \geq k_{0} \forall n>f(k) \quad x \in U_{n}^{k} .
$$

For each $k$, pick $n_{k} \in I_{k}$ such that $n_{k}>f(k)$.

We claim that $\left\{U_{n_{k}}^{k}: k \in \omega\right\}$ is a point-cofinite cover of $X_{\mathfrak{b}}$ : If $x \in X_{\alpha}$, then $x \in U_{n_{k}}^{k}$ for all but finitely many $k$, because $n_{k}>f(k)$ for all $k$. If $x=x_{\beta}$, $\beta \geq \alpha$, then $x \subseteq^{*} x_{\alpha}$. For each large enough $k, m_{n_{k}}^{k}$ is large enough, so that $x \cap\left(m_{n_{k}}^{k}, m_{n_{k}+1}^{k}\right) \subseteq x_{\alpha} \cap\left(m_{n_{k}}^{k}, m_{n_{k}+1}^{k}\right)=\emptyset$, and thus $x \in U_{n_{k}}^{k}$.

Remark 2.6. Zdomskyy points out that for the proof to go through, it suffices that $\left\{x_{\alpha}: \alpha<\mathfrak{b}\right\}$ is such that there is an unbounded $\left\{y_{\alpha}: \alpha<\mathfrak{b}\right\} \subseteq[\omega]^{\omega}$ such that for each $\alpha, x_{\alpha}$ is a pseudointersection of $\left\{y_{\beta}: \beta<\alpha\right\}$. We do not know whether the assertion mentioned here is weaker than the existence of an unbounded tower.

We now turn to nontrivial examples of sets satisfying $S_{1}(\Gamma, \Gamma)$ in all finite powers. In general, $\mathrm{S}_{1}(\Gamma, \Gamma)$ is not preserved by taking finite powers [9], and we use a slightly stronger hypothesis in our construction.

Definition 2.7. Let $\mathfrak{b}_{0}$ be the additivity number of $S_{1}(\Gamma, \Gamma)$, that is, the minimum cardinality of a family $\mathcal{F}$ of sets of reals, each satisfying $S_{1}(\Gamma, \Gamma)$, such that the union of all members of $\mathcal{F}$ does not satisfy $\mathrm{S}_{1}(\Gamma, \Gamma)$.

$\mathfrak{t} \leq \mathfrak{h}$, and Scheepers proved that $\mathfrak{h} \leq \mathfrak{b}_{0} \leq \mathfrak{b}$ [17. It follows from Theorem 3.6 that, consistently, $\mathfrak{h}<\mathfrak{b}_{0}=\mathfrak{b}$. It is open whether $\mathfrak{b}_{0}=\mathfrak{b}$ is provable. If $\mathfrak{t}=\mathfrak{b}$ or $\mathfrak{h}=\mathfrak{b}<\mathfrak{d}$, then there is an unbounded tower of cardinality $\mathfrak{b}_{0}$.

Theorem 2.8. For each unbounded tower $T$ of cardinality $\mathfrak{b}_{0}$, all finite powers of $T \cup[\omega]<\omega$ satisfy $\mathrm{S}_{1}(\Gamma, \Gamma)$.

Proof. We say that $\mathcal{U}$ is an $\omega$-cover of $X$ if no member of $\mathcal{U}$ contains $X$ as a subset, but each finite subset of $X$ is contained in some member of $\mathcal{U}$. We need a multidimensional version of Lemma 1.2 of [6].

Lemma 2.9. Assume that $[\omega]^{<\omega} \subseteq X \subseteq P(\omega)$, and let $e \in \omega$. For each open $\omega$-cover $\mathcal{U}$ of $X^{e}$, there are $m_{0}<m_{1}<\ldots$ and $U_{0}, U_{1}, \cdots \in \mathcal{U}$, such that for all $x_{0}, \ldots, x_{e-1} \subseteq \omega,\left(x_{0}, \ldots, x_{e-1}\right) \in U_{n}$ whenever $x_{i} \cap\left(m_{n}, m_{n+1}\right)=\emptyset$ for all $i<e$. 
Proof. As $\mathcal{U}$ is an open $\omega$-cover of $X^{e}$, there is an open $\omega$-cover $\mathcal{V}$ of $X$ such that $\left\{V^{e}: V \in \mathcal{V}\right\}$ refines $\mathcal{U}[9$.

Let $m_{0}=0$. For each $n \geq 0$ : Assume that $V_{0}, \ldots, V_{n-1} \in \mathcal{V}$ are given, and $U_{0}, \ldots, U_{n-1} \in \mathcal{U}$ are such that $V_{i}^{e} \subseteq U_{i}$ for all $i<n$. Fix a finite $F \subseteq X$ such that $F^{e}$ is not contained in any of the sets $U_{0}, \ldots, U_{n-1}$. As $\mathcal{V}$ is an $\omega$-cover of $X$, there is $V_{n} \in \mathcal{V}$ such that $F \cup P\left(\left\{0, \ldots, m_{n}\right\}\right) \subseteq V_{n}$. Take $U_{n} \in \mathcal{U}$ such that $V_{n}^{e} \subseteq U_{n}$. Then $U_{n} \notin\left\{U_{0}, \ldots, U_{n-1}\right\}$. As $V_{n}$ is open, for each $s \subseteq\left\{0, \ldots, m_{n}\right\}$ there is $k_{s}$ such that for each $x \in P(\omega)$ with $x \cap\left\{0, \ldots, k_{s}-1\right\}=s, x \in V_{n}$. Let $m_{n+1}=\max \left\{k_{s}: s \subseteq\left\{0, \ldots, m_{n}\right\}\right\}$.

If $x_{i} \cap\left(m_{n}, m_{n+1}\right)=\emptyset$ for all $i<e$, then $\left(x_{0}, \ldots, x_{e-1}\right) \in V_{n}^{e} \subseteq U_{n}$.

The assumption in the theorem that there is an unbounded tower of cardinality $\mathfrak{b}_{0}$ implies that $\mathfrak{b}_{0}=\mathfrak{b}$. The proof is by induction on the power $e$ of $T \cup[\omega]<\omega$. The case $e=1$ follows from Theorem 2.5.

Let $\mathcal{U}_{0}, \mathcal{U}_{1}, \cdots \in \Gamma\left(\left(T \cup[\omega]^{<\omega}\right)^{e}\right)$. We may assume that these covers are countable. As in the proof of Theorem 2.5 (this time using Lemma 2.9), there are for each $k$, $m_{0}^{k}<m_{1}^{k}<\ldots$ and $U_{0}^{k}, U_{1}^{k}, \cdots \in \mathcal{U}_{k}$ (so that $\left\{U_{n}^{k}: n \in \omega\right\} \in \Gamma\left(\left(T \cup[\omega]^{<\omega}\right)^{e}\right)$ ), such that for all $y_{0}, \ldots, y_{e-1} \subseteq \omega,\left(y_{0}, \ldots, y_{e-1}\right) \in U_{n}^{k}$ whenever $y_{i} \cap\left(m_{n}^{k}, m_{n+1}^{k}\right)=\emptyset$ for all $i<e$.

Let $\alpha_{0}$ be such that $X_{\alpha_{0}}^{e}$ is not contained in any member of $\bigcup_{n} \mathcal{U}_{n}$. As $T$ is unbounded, there is $\alpha$ such that $\alpha_{0} \leq \alpha<\mathfrak{b}$, and for each $k, I_{k}=\left\{n: x_{\alpha} \cap\right.$ $\left.\left(m_{n}^{k}, m_{n+1}^{k}\right)=\emptyset\right\}$ is infinite.

Let $Y=\left\{x_{\beta}: \beta \geq \alpha\right\}$. $\left(T \cup[\omega]^{<\omega}\right)^{e} \backslash Y^{e}$ is a union of fewer than $\mathfrak{b}_{0}$ homeomorphic copies of $\left(T \cup[\omega]^{<\omega}\right)^{e-1}$. By the induction hypothesis, $\left(T \cup[\omega]^{<\omega}\right)^{e-1}$ satisfies $\mathrm{S}_{1}(\Gamma, \Gamma)$, and therefore so does $\left(T \cup[\omega]^{<\omega}\right)^{e} \backslash Y^{e}$. For each $k,\left\{U_{n}^{k}: n \in I_{k}\right\}$ is a pointcofinite cover of $\left(T \cup[\omega]^{<\omega}\right)^{e} \backslash Y^{e}$, and thus there are infinite $J_{0} \subseteq I_{0}, J_{1} \subseteq I_{1}, \ldots$, such that $\left\{\bigcap_{n \in J_{k}} U_{n}^{k}: k \in \omega\right\}$ is a point-cofinite cover of $\left.\left(T \cup[\omega]^{<\omega}\right)^{e} \backslash Y^{e}\right]$ For each $k$, pick $n_{k} \in J_{k}$ such that: $m_{n_{k}}^{k}>m_{n_{k-1}+1}^{k-1}, x_{\alpha} \cap\left(m_{n_{k}}^{k}, m_{n_{k}+1}^{k}\right)=\emptyset$, and $U_{n_{k}}^{k} \notin\left\{U_{n_{0}}^{0}, \ldots, U_{n_{k-1}}^{k-1}\right\}$.

$\left\{U_{n_{k}}^{k}: k \in \omega\right\} \in \Gamma\left(T \cup[\omega]^{<\omega}\right):$ If $x \in\left(T \cup[\omega]^{<\omega}\right)^{e} \backslash Y^{e}$, then $x \in U_{n_{k}}^{k}$ for all but finitely many $k$. If $x=\left(x_{\beta_{0}}, \ldots, x_{\beta_{e-1}}\right) \in Y$, then $\beta_{0}, \ldots, \beta_{e-1} \geq \alpha$, and thus $x_{\beta_{0}}, \ldots, x_{\beta_{e-1}} \subseteq^{*} x_{\alpha}$. For each large enough $k, m_{n_{k}}^{k}$ is large enough, so that $x_{\beta_{i}} \cap\left(m_{n_{k}}^{k}, m_{n_{k}+1}^{k}\right) \subseteq x_{\alpha} \cap\left(m_{n_{k}}^{k}, m_{n_{k}+1}^{k}\right)=\emptyset$ for all $i<e$, and thus $x \in U_{n_{k}}^{k}$.

There is an additional way to obtain nontrivial $S_{1}(\Gamma, \Gamma)$ sets: The hypothesis $\mathfrak{b}=$ $\operatorname{cov}(\mathcal{N})=\operatorname{cof}(\mathcal{N})$ provides $\mathfrak{b}$-Sierpiński sets, and $\mathfrak{b}$-Sierpiński sets satisfy $\mathrm{S}_{1}(\Gamma, \Gamma)$, even for Borel point-cofinite covers. Details are available in 18 .

We record the following consequence of Theorem 2.3 for later use.

Corollary 2.10. For each unbounded tower $T$ of cardinality $\mathfrak{b}, T \cup[\omega]<\omega$ satisfies $\mathrm{S}_{1}(\Gamma, \Gamma)$ for open covers, but not for Borel covers.

Proof. The latter property is hereditary for subsets [18. By a theorem of Hurewicz, a set of reals satisfies $\mathrm{U}_{\text {fin }}(\Gamma, \Gamma)$ if and only if each continuous image of $X$ in $\omega^{\omega}$ is bounded. It follows that the set $T \subseteq T \cup[\omega]^{<\omega}$ does not even satisfy $\mathrm{U}_{\text {fin }}(\Gamma, \Gamma)$.

\footnotetext{
${ }^{3}$ Choosing infinitely many elements from each cover, instead of one, can be done by adding to the given sequence of covers all cofinite subsets of the given covers.
} 


\section{A CONSISTEnCy RESUlt}

By the results of the previous section, we have the following.

Lemma 3.1. Assume that every set of reals with property $\mathrm{S}_{1}(\Gamma, \Gamma)$ has cardinality $<\mathfrak{b}$, and $\mathfrak{c}=\aleph_{2}$. Then $\aleph_{1}=\mathfrak{t}=\operatorname{cov}(\mathcal{N})<\mathfrak{b}=\aleph_{2}$.

Proof. As there is no unbounded tower, we have that $\mathfrak{t}<\mathfrak{b}=\mathfrak{d}$. As $\mathfrak{c}=\aleph_{2}$, $\aleph_{1}=\mathfrak{t}<\mathfrak{b}=\aleph_{2}$. Since there are no $\mathfrak{b}$-Sierpiński sets and $\mathfrak{b}=\operatorname{cof}(\mathcal{N})=\mathfrak{c}$, $\operatorname{cov}(\mathcal{N})<\mathfrak{b}$.

In Laver's model [1], $\aleph_{1}=\mathfrak{t}=\operatorname{cov}(\mathcal{N})<\mathfrak{b}=\aleph_{2}$. We will show that, indeed, $\mathrm{S}_{1}(\Gamma, \Gamma)$ is trivial there. Laver's model was constructed to realize Borel's Conjecture, asserting that "strong measure zero" is trivial. In some sense, $S_{1}(\Gamma, \Gamma)$ is a dual of strong measure zero. For example, the canonical examples of $S_{1}(\Gamma, \Gamma)$ sets are Sierpiński sets, a measure-theoretic object, whereas the canonical examples of strong measure zero sets are Luzin sets, a Baire category theoretic object. More about that can be seen in 18 .

The main technical result of this paper is the following.

Theorem 3.2. In the Laver model, if $X \subseteq 2^{\omega}$ has cardinality $\mathfrak{b}$, then there is a Borel map $f: 2^{\omega} \rightarrow \omega^{\omega}$ such that $f[X]$ is unbounded.

Proof. The notation in this proof is as in Laver [1]. We will use the following slightly simplified version of Lemma 14 of [11].

Lemma 3.3 (Laver). Let $\mathbb{P}_{\omega_{2}}$ be the countable support iteration of Laver forcing, $p \in \mathbb{P}_{\omega_{2}}$, and let $\stackrel{\circ}{a}$ be $a \mathbb{P}_{\omega_{2}}$-name such that

$$
p \Vdash \stackrel{\circ}{a} \in 2^{\omega} .
$$

Then there are a condition $q$ stronger than $p$ and finite $U_{s} \subseteq 2^{\omega}$ for each $s \in q(0)$ extending the root of $q(0)$ such that for all such $s$ and all $n$ :

$$
q(0)_{t} \wedge q \uparrow\left[1, \omega_{2}\right) \Vdash “ \exists u \in \check{U}_{s} u \uparrow n=\stackrel{\circ}{\uparrow} n \text { " }
$$

for all but finitely many immediate successors $t$ of $s$ in $q(0)$.

Assume that $X \subseteq 2^{\omega}$ has no unbounded Borel image in $\mathcal{M}\left[G_{\omega_{2}}\right]$, i.e., Laver's model. For every code $u \in 2^{\omega}$ for a Borel function $f: 2^{\omega} \rightarrow \omega^{\omega}$ there exists $g \in \omega^{\omega}$ such that for every $x \in X$ we have that $f(x) \leq^{*} g$.

By a standard Löwenheim-Skolem argument (see Theorem 4.5 on page 281 of [3] or section 4 on page 580 of [12]), we may find $\alpha<\omega_{2}$ such that for every code $u \in \mathcal{M}\left[G_{\alpha}\right]$ there is an upper bound $g \in \mathcal{M}\left[G_{\alpha}\right]$. By the arguments employed by Laver [11, Lemmata 10 and 11], we may assume that $\mathcal{M}\left[G_{\alpha}\right]$ is the ground model $\mathcal{M}$.

Since the continuum hypothesis holds in $\mathcal{M}$ and $|X|=\mathfrak{b}=\aleph_{2}$, there are $p \in G_{\omega_{2}}$ and $\stackrel{\circ}{a}$ such that

$$
p \Vdash \stackrel{\circ}{a} \in \stackrel{\circ}{X} \text { and } \stackrel{\circ}{a} \notin \mathcal{M} .
$$

Work in the ground model $\mathcal{M}$.

Let $q \leq p$ be as in Lemma 3.3. Define

$$
Q=\{s \in q(0): \operatorname{root}(q(0)) \subseteq s\}
$$

and let $U_{s}, s \in Q$, be the finite sets from the lemma. Let $U=\bigcup_{s \in Q} U_{s}$. Define a Borel map $f: 2^{\omega} \rightarrow \omega^{Q}$ so that for every $x \in 2^{\omega} \backslash U$ and for each $s \in Q$ : If 
$f(x)(s)=n$, then $x\left\lceil n \neq u\left\lceil n\right.\right.$ for each $u \in U_{s}$. For $x \in U, f(x)$ may be arbitrary. There must be a $g \in \omega^{Q} \cap \mathcal{M}$ and $r \leq q$ such that

$$
r \Vdash f(\stackrel{\circ}{a}) \leq^{*} \check{g} .
$$

Since $p$ forced that $a$ is not in the ground model, it cannot be that $a$ is in $U$. We may extend $r(0)$ if necessary so that if $s=\operatorname{root}(r(0))$, then

$$
r \Vdash f(\stackrel{\circ}{a})(s) \leq \check{g}(s) .
$$

But this is a contradiction to Lemma 3.3 since for all but finitely many $t \in r(0)$ which are immediate extensions of $s$ :

$$
r(0)_{t} \wedge q\left\lceil\left[1, \omega_{2}\right) \Vdash f(\stackrel{\circ}{a})(s)>\check{g}(s) .\right.
$$

In 20, Tsaban and Zdomskyy prove that $S_{1}(\Gamma, \Gamma)$ for Borel covers is equivalent to the Kočinac property $S_{\text {cof }}(\Gamma, \Gamma)\left[10\right.$, asserting that for all $\mathcal{U}_{0}, \mathcal{U}_{1}, \cdots \in \Gamma$, there are cofinite subsets $\mathcal{V}_{0} \subseteq \mathcal{U}_{0}, \mathcal{V}_{1} \subseteq \mathcal{U}_{1}, \ldots$ such that $\bigcup_{n} \mathcal{V}_{n} \in \Gamma$. The main result of [5] can be reformulated as follows.

Theorem 3.4 (Dow [5]). In Laver's model, $\mathrm{S}_{1}(\Gamma, \Gamma)$ implies $\mathrm{S}_{\text {cof }}(\Gamma, \Gamma)$.

For the reader's convenience, we give Dow's proof, adapted to the present notation.

Proof. A family $\mathcal{H} \subseteq[\omega]^{\omega}$ is $\omega$-splitting if for each countable $\mathcal{A} \subseteq[\omega]^{\omega}$, there is $H \in \mathcal{H}$ which splits each element of $\mathcal{A}$, i.e.,

$$
|A \cap H|=|A \backslash H|=\omega \text { for all } A \in \mathcal{A} .
$$

The main technical result in [5] is the following.

Lemma 3.5 (Dow). In Laver's model, each $\omega$-splitting family contains an $\omega$ splitting family of cardinality $<\mathfrak{b}$.

Assume that $X$ satisfies $\mathrm{S}_{1}(\Gamma, \Gamma)$. Let $\mathcal{U}_{0}, \mathcal{U}_{1}, \ldots$ be open point-cofinite countable covers of $X$. We may assume that $\mathcal{U}_{i} \cap \mathcal{U}_{j}=\emptyset$ whenever $i \neq j$. Put $\mathcal{U}=\bigcup_{n<\omega} \mathcal{U}_{n}$. We identify $\mathcal{U}$ with $\omega$, its cardinality.

Define $\mathcal{H} \subseteq[\mathcal{U}]^{\omega}$ as follows. For $H \in[\mathcal{U}]^{\omega}$, put $H \in \mathcal{H}$ if and only if there exists $\mathcal{V} \in[\mathcal{U}]^{\omega}$, a point-cofinite cover of $X$, such that $H \cap \mathcal{U}_{n} \subseteq^{*} \mathcal{V}$ for all $n$. We claim that $\mathcal{H}$ is an $\omega$-splitting family. As $\mathcal{H}$ is closed under taking infinite subsets, it suffices to show that it is $\omega$-hitting; i.e., for any countable $\mathcal{A} \subseteq[\mathcal{U}]^{\omega}$ there exists $H \in \mathcal{H}$ which intersects each $A \in \mathcal{A}$. (It is enough to intersect each $A \in \mathcal{A}$, since we may assume that $\mathcal{A}$ is closed under taking cofinite subsets.)

Let $\mathcal{A} \subseteq[\mathcal{U}]^{\omega}$ be countable. For each $n$, choose sets $\mathcal{U}_{n, m} \in\left[\mathcal{U}_{n}\right]^{\omega}, m \in \omega$, such that for each $A \in \mathcal{A}$, if $A \cap \mathcal{U}_{n}$ is infinite, then $\mathcal{U}_{n, m} \subseteq A$ for some $m$. Apply the $\mathrm{S}_{1}(\Gamma, \Gamma)$ to the family $\left\{\mathcal{U}_{n, m}: n, m \in \omega\right\}$ to obtain a point-cofinite $\mathcal{V} \subseteq \mathcal{U}$ such that $\mathcal{V} \cap \mathcal{U}_{n, m}$ is nonempty for all $n, m$.

Next, choose finite subsets $\mathcal{F}_{n} \subseteq \mathcal{U}_{n}, n \in \omega$, such that for each $A \in \mathcal{A}$ with $A \cap \mathcal{U}_{n}$ finite for all $n$, then $A \subseteq^{*} \bigcup_{n} \mathcal{F}_{n}$. Take $H=\mathcal{V} \cup \bigcup_{n} \mathcal{F}_{n}$. Then $H$ is in $\mathcal{H}$ and meets each $A \in \mathcal{A}$. This shows that $\mathcal{H}$ is an $\omega$-splitting family.

By Lemma 3.5, there is an $\omega$-splitting $\mathcal{H}^{\prime} \subseteq \mathcal{H}$ of cardinality $<\mathfrak{b}$. For each $H \in \mathcal{H}^{\prime}$, let $\mathcal{V}_{H}$ witness that $H$ is in $\mathcal{H}$; i.e., $\mathcal{V}_{H} \subseteq \mathcal{U}$ is a point-cofinite cover of $X$ and $H \cap \mathcal{U}_{n} \subseteq^{*} \mathcal{V}_{H}$ for all $n$.

\footnotetext{
${ }^{4}$ To see why, replace each $\mathcal{U}_{n}$ by $\mathcal{U}_{n} \backslash \bigcup_{i<n} \mathcal{U}_{i}$ and discard the finite ones. It suffices to show that $\mathrm{S}_{\mathrm{cof}}(\Gamma, \Gamma)$ applies to those that are left.
} 
By the definition of $\mathfrak{b}$, we may find finite $\mathcal{F}_{n} \subseteq \mathcal{U}_{n}, n \in \omega$, such that for each $H \in \mathcal{H}^{\prime}$,

$$
H \cap \mathcal{U}_{n} \subseteq \mathcal{V}_{H} \cup \mathcal{F}_{n}
$$

for all but finitely many $n$. We claim that $\mathcal{W}=\bigcup_{n} \mathcal{U}_{n} \backslash \mathcal{F}_{n}$ is point-cofinite. Suppose it is not. Then there is $x \in X$ such that for infinitely many $n$, there is $U_{n} \in \mathcal{U}_{n} \backslash \mathcal{F}_{n}$ with $x \notin U_{n}$. Let $H \in \mathcal{H}^{\prime}$ contain infinitely many of these $U_{n}$. By the above inclusion, all but finitely many of these $U_{n}$ are in $\mathcal{V}_{H}$. This contradicts the fact that $\mathcal{V}_{H}$ is point-cofinite.

We therefore have the following.

Theorem 3.6. In Laver's model, each set of reals $X$ satisfying $\mathrm{S}_{1}(\Gamma, \Gamma)$ has cardinality less than $\mathfrak{b}$.

Proof. By Dow's Theorem, $S_{1}(\Gamma, \Gamma)$ implies $S_{\text {cof }}(\Gamma, \Gamma)$, which in turn implies $\mathrm{S}_{1}(\Gamma, \Gamma)$ for Borel covers [20]. The latter property is equivalent to having all Borel images in $\omega^{\omega}$ bounded [18]. Apply Theorem 3.2.

Thus, it is consistent that strong measure zero and $S_{1}(\Gamma, \Gamma)$ are both trivial.

The proof of Dow's Theorem 3.4 becomes more natural after replacing, in Lemma [3.5 " $\omega$-splitting" by " $\omega$-hitting". This is possible, due to the following fact (cf. Remark 4 of [5]).

Proposition 3.7. For each infinite cardinal $\kappa$, the following are equivalent:

(1) Each $\omega$-splitting family contains an $\omega$-splitting family of cardinality $<\kappa$.

(2) Each $\omega$-hitting family contains an $\omega$-hitting family of cardinality $<\kappa$.

Proof. $(1 \Rightarrow 2)$ Suppose $\mathcal{A}$ is an $\omega$-hitting family. Let $\mathcal{B}=\bigcup_{A \in \mathcal{A}}[A]^{\omega}$. Then $\mathcal{B}$ is $\omega$-splitting. By (1) there exists $\mathcal{C} \subseteq \mathcal{B}$ of size $<\kappa$ which is $\omega$-splitting. Choose $\mathcal{D} \subseteq \mathcal{A}$ of size $<\kappa$ such that for every $C \in \mathcal{C}$ there exists $D \in \mathcal{D}$ with $C \subseteq D$. Then $\mathcal{D}$ is $\omega$-hitting.

$(2 \Rightarrow 1)$ Suppose $\mathcal{A}$ is an $\omega$-splitting family. For each $A \subseteq \omega$ define

$$
A^{*}=\{2 n: n \in A\} \cup\{2 n+1: n \in \bar{A}\} .
$$

Then the family $\mathcal{A}^{*}=\left\{A^{*}: A \in \mathcal{A}\right\}$ is $\omega$-hitting. To see this, suppose that $\mathcal{B}$ is countable. Without loss we may assume that $\mathcal{B}=\mathcal{B}_{0} \cup \mathcal{B}_{1}$, where each element of $\mathcal{B}_{0}$ is a subset of the evens and each element of $\mathcal{B}_{1}$ is a subset of the odds. For $B \in \mathcal{B}_{0}$ let $C_{B}=\{n: 2 n \in B\}$ and for $B \in \mathcal{B}_{1}$ let $C_{B}=\{n: 2 n+1 \in B\}$. Now put

$$
\mathcal{C}=\left\{C_{B}: B \in \mathcal{B}\right\}
$$

Since $\mathcal{A}$ is $\omega$-splitting there is $A \in \mathcal{A}$ which splits $\mathcal{C}$. If $B \in B_{0}$, then $A \cap C_{B}$ infinite implies $B \cap A^{*}$ infinite. If $B \in B_{1}$, then $\bar{A} \cap C_{B}$ infinite implies $B \cap A^{*}$ infinite.

By (2) there exists $\mathcal{A}_{0} \subseteq \mathcal{A}$ of cardinality $<\kappa$ such that $\mathcal{A}_{0}^{*}$ is $\omega$-hitting. We claim that $\mathcal{A}_{0}$ is $\omega$-splitting. Given any $B \subseteq \omega$ let $B^{\prime}=\{2 n: n \in B\}$ and let $B^{\prime \prime}=\{2 n+1: n \in B\}$. Given $\mathcal{B} \subseteq[\omega]^{\omega}$ countable, there exists $A \in \mathcal{A}_{0}$ such that $A^{*}$ hits each $B^{\prime}$ and $B^{\prime \prime}$ for $B \in \mathcal{B}$. But this implies that $A$ splits $B$.

\section{Applichtions to Arhangel'skiI's $\alpha_{i}$ SPACES}

Let $Y$ be a general (not necessarily metrizable) topological space. We say that a countably infinite set $A \subseteq Y$ converges to a point $y \in Y$ if each (equivalently, some) bijective enumeration of $A$ converges to $y$. The following concepts are due 
to Arhangel'skii. $Y$ is an $\alpha_{1}$ space if for each $y \in Y$ and each sequence $A_{0}, A_{1}, \ldots$ of countably infinite sets, each converging to $y$, there are cofinite $B_{0} \subseteq A_{0}, B_{1} \subseteq$ $A_{1}, \ldots$, such that $\bigcup_{n} B_{n}$ converges to $y$. Replacing "cofinite" by "singletons" (or equivalently, by "infinite"), we obtain the definition of an $\alpha_{2}$ space.

We first consider countable spaces.

Definition 4.1. Let $X$ be a set of reals, and let $\mathcal{U}_{0}, \mathcal{U}_{1}, \ldots$ be countable pointcofinite covers of $X$. For each $n$, enumerate bijectively $\mathcal{U}_{n}=\left\{U_{m}^{n}: m \in \omega\right\}$. We associate to $X$ a (new) topology $\tau$ on the fan $S_{\omega}=\omega \times \omega \cup\{\infty\}$ as follows: $\infty$ is the only nonisolated point of $S_{\omega}$, and a neighborhood base at $\infty$ is given by the sets

$$
[\infty]_{F}=\left\{(n, m): F \subseteq U_{m}^{n}\right\}
$$

for each finite $F \subseteq X$.

Lemma 4.2. In the notation of Definition 4.1: $A$ converges to $\infty$ in $\tau$ if and only if $\mathcal{U}(A)=\left\{U_{m}^{n}:(n, m) \in A\right\}$ is a point-cofinite cover of $X$.

Assume that there is an unbounded tower. By Corollary 2.10, there is a set of reals $X$ satisfying $\mathrm{S}_{1}(\Gamma, \Gamma)$ but not $\mathrm{S}_{\text {cof }}(\Gamma, \Gamma)$. Let $\mathcal{U}_{0}, \mathcal{U}_{1}, \ldots$ be countable open point-cofinite covers of $X$ witnessing the failure of $\mathrm{S}_{\mathrm{cof}}(\Gamma, \Gamma)$. Then, by Lemma 4.2, $\left(S_{\omega}, \tau\right)$ is $\alpha_{2}$ but not $\alpha_{1}$. In particular, we reproduce the following.

Corollary 4.3 (Nyikos [13]). If there is an unbounded tower of cardinality $\mathfrak{b}$, then there is a countable $\alpha_{2}$ space which is not an $\alpha_{1}$ space.

Recall that by Proposition 2.4 it suffices to assume in Corollary 4.3 the existence of any unbounded tower.

Next, we consider spaces of continuous functions. Consider $C(X)$, the family of continuous real-valued functions, as a subspace of the Tychonoff product $\mathbb{R}^{X}$, i.e., with the topology of pointwise convergence. Sakai [14] proved that $X$ satisfies $\mathrm{S}_{1}(\Gamma, \Gamma)$ for clopen covers if and only if $C(X)$ is an $\alpha_{2}$ space. The main result of 20. is that $C(X)$ is $\alpha_{1}$ if and only if $X$ satisfies $\mathrm{S}_{1}(\Gamma, \Gamma)$ for Borel covers (equivalently, each Borel image of $X$ in $\omega^{\omega}$ is bounded).

The Scheepers Conjecture is that for subsets of $\mathbb{R} \backslash \mathbb{Q}, \mathrm{S}_{1}(\Gamma, \Gamma)$ for clopen covers implies $S_{1}(\Gamma, \Gamma)$ for open covers. Dow [5] proved that in Laver's model, every $\alpha_{2}$ space is $\alpha_{1}$. By Theorem 3.2, we can add the last item in the following list.

Corollary 4.4. In Laver's model, the following are equivalent for sets of reals $X$ :

(1) $C(X)$ is an $\alpha_{2}$ space;

(2) $C(X)$ is an $\alpha_{1}$ space;

(3) $X$ satisfies $\mathrm{S}_{1}(\Gamma, \Gamma)$ for clopen covers;

(4) $X$ satisfies $\mathrm{S}_{1}(\Gamma, \Gamma)$ for open covers;

(5) $X$ satisfies $\mathrm{S}_{1}(\Gamma, \Gamma)$ for Borel covers;

(6) $|X|<\mathfrak{b}$.

On the other hand, Corollary 2.10 implies the following.

Corollary 4.5. If there is an unbounded tower, then there is a set of reals $X$ such that $C(X)$ is $\alpha_{2}$ but not $\alpha_{1}$.

Essentially, Corollary 4.3 is a special case of Corollary 2.10, whereas Corollary 4.5 is equivalent to Corollary 2.10 . 


\section{ACKNOWLEDGMENT}

The authors thank Lyubomyr Zdomskyy for his useful comments.

\section{REFERENCES}

[1] T. Bartoszyński and S. Shelah, Continuous images of sets of reals, Topology and its Applications 116 (2001), 243-253. MR.1855966 (2002f:03090)

[2] T. Bartoszyński and B. Tsaban, Hereditary topological diagonalizations and the MengerHurewicz Conjectures, Proceedings of the American Mathematical Society, 134 (2006), 605615. MR2176030 (2006f:54038)

[3] J. Baumgartner and R. Laver, Iterated perfect-set forcing, Annals of Mathematical Logic 17 (1979), 271-288. MR556894(81a:03050)

[4] A. Blass, Combinatorial cardinal characteristics of the continuum, in: Handbook of Set Theory (M. Foreman, A. Kanamori, and M. Magidor, eds.), Kluwer Academic Publishers, Dordrecht, to appear. http://www.math.lsa.umich.edu/ ablass/hbk.pdf

[5] A. Dow, Two classes of Fréchet-Urysohn spaces, Proceedings of the American Mathematical Society 108 (1990), 241-247. MR975638 (90j:54028)

[6] F. Galvin and A. Miller, $\gamma$-sets and other singular sets of real numbers, Topology and its Applications 17 (1984), 145-155. MR738943 (85f:54011)

[7] J. Gerlits and Zs. Nagy, Some properties of $C(X)$. I, Topology and its Applications 14 (1982), 151-161. MR667661 (84f:54021)

[8] W. Hurewicz, Uber eine Verallgemeinerung des Borelschen Theorems, Mathematische Zeitschrift 24 (1925), 401-421.

[9] W. Just, A. Miller, M. Scheepers, and P. Szeptycki, The combinatorics of open covers. II, Topology and its Applications 73 (1996), 241-266. MR1419798 (98g:03115a)

[10] L. Kočinac, Selection principles related to $\alpha_{i}$-properties, Taiwanese Journal of Mathematics 12 (2008), 561-572. MR2417134 (2009h:91049)

[11] R. Laver, On the consistency of Borel's conjecture, Acta Mathematica 137 (1976), 151-169. MR0422027 (54:10019)

[12] A. Miller, Mapping a set of reals onto the reals, Journal of Symbolic Logic 48 (1983), 575-584. MR716618 (84k:03125)

[13] P. Nyikos, Subsets of $\omega^{\omega}$ and the Fréchet-Urysohn and $\alpha_{i}$-properties, Topology and its Applications 48 (1992), 91-116. MR1195504 (93k:54011)

[14] M. Sakai, The sequence selection properties of $C_{p}(X)$, Topology and its Applications 154 (2007), 552-560. MR2280899 (2007k:54007)

[15] M. Scheepers, Combinatorics of open covers. I: Ramsey theory, Topology and its Applications 69 (1996), 31-62. MR1378387 (97h:90123)

[16] M. Scheepers, $C_{p}(X)$ and Arhangel'skiu's $\alpha_{i}$ spaces, Topology and its Applications 89 (1998), 265-275. MR1645184 (99g:54018)

[17] M. Scheepers, Sequential convergence in $\mathrm{C}_{p}(X)$ and a covering property, East-West Journal of Mathematics 1 (1999), 207-214. MR1727383 (2000i:54014)

[18] M. Scheepers and B. Tsaban, The combinatorics of Borel covers, Topology and its Applications 121 (2002), 357-382. MR1908999 (2003e:03091)

[19] B. Tsaban, Menger's and Hurewicz's Problems: Solutions from "The Book" and refinements, Contemporary Mathematics, American Mathematical Society, to appear.

[20] B. Tsaban and L. Zdomskyy, Hereditarily Hurewicz spaces and Arhangel'skiu sheaf amalgamations, Journal of the European Mathematical Society, to appear.

Department of Mathematics, University of Wisconsin-Madison, Van Vleck Hall, 480 Lincoln Drive, Madison, Wisconsin 53706-1388

E-mail address: miller@math.wisc.edu

URL: http://www.math.wisc.edu/ miller/

Department of Mathematics, Bar-Ilan University, Ramat-Gan 52900, Israel

E-mail address: tsaban@math.biu.ac.il

$U R L$ : http://www.cs.biu.ac.il/ tsaban 